\title{
Supermassive Black Hole Growth and Merger Rates from Cosmological N-body Simulations
}

\author{
Miroslav Micic ${ }^{1 \star}$, Kelly Holley-Bockelmann ${ }^{2}$, Steinn Sigurdsson ${ }^{1}$ \& Tom Abel ${ }^{3}$ \\ ${ }^{1}$ Department of Astronomy \& Astrophysics, Pennsylvania State University \\ 2 IGPG, Pennsylvania State University \\ 3 SLAC, Stanford University
}

22 March 2007

\begin{abstract}
Understanding how seed black holes grow into intermediate and supermassive black holes (IMBHs and SMBHs, respectively) has important implications for the duty-cycle of active galactic nuclei (AGN), galaxy evolution, and gravitational wave astronomy. Most studies of the cosmological growth and merger history of black holes have used semianalytic models and have concentrated on SMBH growth in luminous galaxies. Using high resolution cosmological N-body simulations, we track the assembly of black holes over a large range of final masses - from seed black holes to SMBHs over widely varying dynamical histories. We used the dynamics of dark matter halos to track the evolution of seed black holes in three different gas accretion scenarios. We have found that growth of a Sagittarius $A^{*}$ - size SMBH reaches its maximum mass $\mathrm{M}_{\mathrm{SMBH}}=\sim 10^{6} \mathrm{M}_{\odot}$ at $\mathrm{z} \sim 6$ through early gaseous accretion episodes, after which it stays at near constant mass. At the same redshift, the duty-cycle of the host AGN ends, hence redshift $\mathrm{z}=6$ marks the transition from an AGN to a starburst galaxy which eventually becomes the Milky Way. By tracking black hole growth as a function of time and mass, we estimate that the IMBH merger rate reaches a maximum of $\mathrm{R}_{\max }=55 \mathrm{yr}^{-1}$ at $\mathrm{z}=11$. From IMBH merger rates we calculate $\mathrm{N}_{\mathrm{ULX}}=7$ per Milky Way type galaxy per redshift in redshift range $2 \lesssim z \lessgtr 6$.
\end{abstract}

Key words:

stars: Population III, intermediate mass black holes, supermassive black holes, gravitational waves, dark matter halos

\section{INTRODUCTION}

Supermassive black holes (SMBH) are thought to dwell at the centers of most galaxies, (Kormendy \& Richstone 1995) with masses between $10^{6} \mathrm{M}_{\odot} \lesssim \mathrm{M} \lesssim 10^{9} \mathrm{M}_{\odot}$. In principle, the abundance of SMBHs today can be explained if they grow through mergers and early accretion (Schneider et al. 2002), from a gaseous disk. The most likely candidates for SMBH seeds are black holes that form as remnants of Population III stars at redshifts z $\gtrsim 12-20$ (Heger et al. 2003, Volonteri et al. 2003, Islam et al. 2003, Wise \& Abel 2005). These relic seeds are predicted to form in the centers of dark matter halos (DMH), and have masses $\lesssim 10^{3} \mathrm{M}_{\odot}$ (Abel et al. 2000, 2002). DMHs form in the early universe and hierarchically merge into larger bound objects. As DMHs merge into massive halos, the seed black holes sink to the center through dynamical friction and eventually coalesce.

Although the seed formation stops at z 12 as Population III supernovae rates drop to zero (Wise \& Abel 2005), SMBH growth continues as DMH mergers proceed to low redshifts. From a combination of gas accretion and binary black hole coalescence, seeds can grow to intermediate mass black holes (IMBHs, with masses $10^{2} \mathrm{M}_{\odot} \lesssim \mathrm{m} \lesssim 10^{5} \mathrm{M}_{\odot}$ ). With continued mergers and gas accretion, it is thought that these IMBHs may form the SMBHs we observe today. The detection of IMBHs

^ E-mail: micic@astro.psu.edu, kellyhb@gravity.psu.edu, steinn@astro.psu.edu, tabel@slac.stanford.edu 


\section{Miroslav Micic, Kelly Holley-Bockelmann, Steinn Sigurdsson, Tom Abel}

is a matter of debate, but possible candidates are ultraluminous X-ray sources in young star-forming regions (Fabbiano 1989, Roberts \& Warwick 2000, Ptak \& Colbert 2004, Fabbiano \& White 2006) and nearby extragalactic star clusters.

Although this scenario works in general, real detailed understanding of how seed black holes grow is a remaining challenge. For example, there are still debates on the seed black hole mass, on the mass of halos that can host seeds, on the seed formation redshift, on the type and efficiency of gas accretion, and on the dynamics of the black hole mergers, all of which compound to yield a huge range in black hole merger rate estimates. Recent black hole merger rate calculations span over three orders of magnitude: Haehnelt (1994) calculates the merger rate to be $\mathrm{R} \sim 0.1$ - 100 events per year; Menou et al. (2001), R 1 - 100 $\mathrm{yr}^{-1}$; Wyithe \& Loeb (2003), R $15-350 \mathrm{yr}^{-1}$; Sesana et al. (2004), R $10 \mathrm{yr}^{-1}$; and Rhook \& Wyithe (2005), R $15 \mathrm{yr}^{-1}$.

There are two general approaches to the problem: direct cosmological N-body simulations and analytical techniques based on a Press-Schechter (PS) formalism (Press \& Schechter 1974). Both methods extract merger rates from the DMHs' merger tree. N-body simulations have the advantage that the evolution of density fluctuations is followed in complete generality, without the need for any of the assumptions involved in creating PS merger trees. Extended Press-Schechter theory (EPS) combines the PS halo mass function with halo merger rates derived by Lacey \& Cole (1993), and it stands as the most widely used method for calculating merger rates. Unfortunately, EPS is mathematically inconsistent (Erickcek et al. 2006) since it provides two equally valid merger rates for the same pair of DMHs. It is also unclear whether EPS reproduces the DMH mass function at high redshifts (Reed et al. 2007). With N-body simulations, there are, in principle, no constraints on the halo mass, structure or kinematics. Unfortunately, the mass and spatial resolution needs to be extremely high, and even in the best resolved N-body simulations of a large volume $\Lambda$ CDM universe (Nagashima et al. 2005), the mass resolution sets the minimum DMH mass to $3 \times 10^{9} \mathrm{M}_{\odot}$ for halos with as few as ten particles. Hence using direct N-body simulations to track the dynamics of seed black holes as they grow within a large comoving volume is out of reach of our current technology.

In this paper, we have developed a hybrid method to follow the merger history of seed black holes as they grow. We performed a high resolution cosmological N-body simulation in the unexplored parameter space of a small cosmological volume but with very high mass resolution to achieve well-resolved halos with a minimum of 32 particles and mass as low as $\mathrm{M}_{\text {halo }}=2.8 \times 10^{7} \mathrm{M}_{\odot}$. The goal was to look at a representative "Local Group" structure, comparable to the one which hosts the Milky Way, and to resolve as low a mass as feasible. We improved the algorithms for identifying dark matter structures and developed set of physically-motivated criteria for seeding DMHs. We then constructed the DMH merger tree, which provides a testbed to study the effects of different gas accretion scenarios. In addition to the merger rate, we extracted observables such as the black hole mass function over cosmological time. This method is a major improvement in calculations of MBH merger rates and presents the first step toward a full treatment of the black hole growth problem. Massive black holes mergers will be one of the prime signals for future space-based gravitational wave observatories like LISA (Laser Interferometer Space Antenna) and BBO (Big Bang Observer). Possible detection depends on a number of parameters: the mass ratio of merging black holes, the total mass of the black hole binary and the redshift.

We describe our simulation and DMH seeding criteria in Section 2; obtaining DMH and black hole merger trees in Section 3 and 4 together with description of black hole growth models; black hole merger rates in Section 5; finally, we discuss our results in Section 6.

\section{SIMULATION}

\subsection{Simulation Setup}

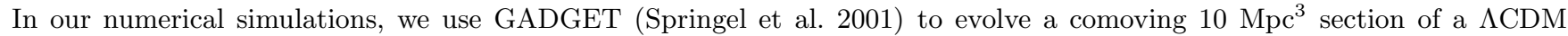
universe $\left(\Omega_{\mathrm{M}}=0.3, \Omega_{\Lambda}=0.7\right.$ and $\left.\mathrm{h}=0.7\right)$ from $z=40$ to $z=0$. We refine a sphere of $2 \mathrm{Mpc}$ in the box to simulate at a higher resolution with $4.9 \times 10^{6}$ high-resolution particles (softening length $2 \mathrm{kpc}$ comoving). The rest of the box has $2.0 \times 10^{6}$ low-resolution particles (softening length $4 \mathrm{kpc}$ comoving). The mass of each high resolution particle in this simulation is $8.85 \times 10^{5} \mathrm{M}_{\odot}$, and the mass of each low-resolution particle is $5.66 \times 10^{7} \mathrm{M}_{\odot}$. A detailed description of this simulation can be found in Micic et al. 2006.

\subsection{Linking Length Problem}

Identifying and following a real $\mathrm{DMH}$ in a cosmological simulation is technically challenging. If the minimum number of particles in a halo is set too low, the DMH identified at one time-step might later disperse. In the widely used Friends-ofFriends algorithm (FOF), if the linking length (b) is too large, large dark matter structures might bridge through a small number of tidally stripped particles. When this occurs, the FOF algorithm can not properly identify DMHs. A linking length of 0.2 has traditionally been used (Davis et al. 1985) since the DMH mass function obtained with it proved to be consistent with observations at low redshifts and mass functions obtained by PS theory. However, very little is known about DMH mass functions at high redshifts $\mathrm{z} \gtrsim 10$ and low masses $\mathrm{M} \lesssim 10^{9} \mathrm{M}_{\odot}$, or about the validity of this linking length. For example, Gao et al. (2005) noted that the first massive halos are found in regions containing many smaller halos which line up along filaments 
and sheets and can not be identified by the FOF algorithm when a linking length of $b=0.2$ is used. The only criterion for initializing linking length comes from the spatial resolution in the performed numerical simulation. The linking length has to be larger than the softening length. High resolution simulations have small softening lengths, which allow the linking length to be smaller as well. Consequently, in our high resolution simulation, we set $b=0.1$. With this value for linking length we eliminated all the problems in identification of dark matter structures.

\subsection{Analysis Tools}

We used P-GroupFinder algorithm (Springel 2000) to identify DMHs in each of our 104 GADGET output files. The analysis starts with a FOF algorithm $(b=0.1)$ which spatially separates dark matter halos. The minimum number of particles per halo is set to 32 . The minimum DMH mass resolved with 32 particles in our simulation is $\mathrm{M}_{\text {halo }}=2.8 \times 10^{7} \mathrm{M}_{\odot}$. Then, we find a gravitationally bound counterpart for each FOF halo and continue with the SUBFIND algorithm to identify substructures in every DMH. We require that halos and subhalos be virialized to be considered real. This requirement is met when the DMH density is above 200 times the average density of the universe at a specific redshift. Since we are studying merger rates for Pop III massive black holes, we then select those halos that are capable of hosting Pop III stars, using primordial supernova rates (Wise \& Abel 2005). Primordial stars can form in DMHs with a minimum mass of $4.16 \times 10^{6} \mathrm{M}_{\odot}$ if $\mathrm{H}_{2}$ cooling is the primary mechanism and if the UV background has a negative feedback. They can also form through hydrogen atomic line cooling in halos with mass $\mathrm{M}_{\mathrm{vir}} \gtrsim 10^{8} \mathrm{M}_{\odot}[(1+\mathrm{z}) / 10]^{-1.5}$. The lifetime of Pop III stars is $\sim 3$ Myrs and the end product is a population of seed black holes with $100 \mathrm{M}_{\odot} \lesssim \mathrm{M}_{\mathrm{BH}} \lesssim 1000 \mathrm{M}_{\odot}$. The first Pop III seed black holes form at redshift $\mathrm{z} \sim 20$ and the last Pop III stars die at redshift $\mathrm{z} \sim 12$. We select the interval $12 \lesssim \mathrm{z} \lesssim 19$ to seed those DMHs which have masses $\mathrm{M}_{\mathrm{vir}} \gtrsim 10^{8} \mathrm{M}_{\odot}[(1+\mathrm{z}) / 10]^{-1.5}$. At $\mathrm{z}=19$ all DMHs with mass above $\mathrm{M}_{\text {halo }}=3.5 \times 10^{7} \mathrm{M}_{\odot}$ and at $\mathrm{z}=12$ all of the DMHs with mass above $\mathrm{M}_{\text {halo }}=6.7 \times 10^{7} \mathrm{M}_{\odot}$ are seeded.

\section{DARK MATTER HALOS' MERGER TREE}

Starting with the first snapshot "i" , we identify DMHs with the set of criteria described above. The most bound particle at the center of each DMH that satisfies the criteria is set to be a seed black hole. At the following snapshot "i+1" , we identify halos from the previous "i" snapshot. At "i+1" we again seed new halos that satisfy our criteria. By "new" halos we consider only those halos that do not have any particles that were part of any already seeded halo. This is because once a halo is seeded it can not form a new black hole due to feedback from the UV background of Pop III stars (Machacek et al. 2001). Meanwhile, we also trace the parent DMH at "i" for every particle in every DMH at "i+1", thus constructing a complete merger history. The method is repeated through the redshift range $12 \lesssim \mathrm{z} \lesssim 19$. Seeding stops at redshift $\mathrm{z}=12 \mathrm{but}$ we continue with creating a merger history until redshift $\mathrm{z}=0$. In total we have 1447 seed black holes.

\section{ASSEMBLING A BLACK HOLE}

After constructing the DMH merger tree, we turn our attention to the evolution of the seeds. The N-body simulation tracks all the seed black holes and their host halos from $\mathrm{z}=19-0$. In order to study black hole growth as a function of time, environment, and merger type, we constructed three black hole merger models which we detail below.

We parameterize the degree of gas accretion involved in growing the black hole and the efficiency of binary coalescence. In the fiducial case, we modeled black hole mergers in the simplest possible way. We assume that the hardening timescale (the time for two black holes to form hard binary) is rapid, that the loss cone is full (Berczik et al. 2006, Sigurdsson 2003, Holley-Bockelmann \& Sigurdsson 2006), that no black holes are ejected, and that there is no gas accretion involved. This gives us a strawman model with which to compare more realistic black hole growth scenarios. We assign seed black hole masses of $200 \mathrm{M}_{\odot}$. When two DMHs merge, their seeds form a binary and merge at the center of the remnant with the total combined mass of its progenitors. If more than two DMHs merge, as often happens early on, the merger is sorted by DMH mass, since dynamical friction is stronger on higher masses. Escala et al. 2006 suggests that the black hole binary will merge within a few times $10^{7}$ yr. Since DMHs typically merge in $10^{8} \mathrm{yr}$, the MBHs will coalesce soon after the DMHs merge in this model. As the halos evolve to the present day, black holes can only grow in mass through mergers, and in our (small cosmological volume) simulation reached a maximum mass of $\mathrm{M}_{\mathrm{BH}}=3 \times 10^{5} \mathrm{M}_{\odot}$. We find that this value is too small to match observations.

For a sustained Eddington accretion, the mass growth rate is:

$\dot{M}_{\mathrm{BH}}=\frac{M_{\mathrm{BH}}}{t_{\mathrm{Sal}}}$

where $\mathrm{t}_{\mathrm{Sal}}$ is the Salpeter time-scale, $\mathrm{t}_{\mathrm{Sal}} \sim 4 \times 10^{7} \mathrm{yr}$ from the recent observations (Hu et al. 2006). In our models, we assume that accretion is triggered by galaxy mergers and lasts $\sim \mathrm{t}_{\text {Sal }}$. During this time, the black holes approximately double their 


\section{Miroslav Micic, Kelly Holley-Bockelmann, Steinn Sigurdsson, Tom Abel}

mass. However, we do not assume that every merger supplies enough gas for the black hole to double its mass. For example, during minor galaxy mergers (mass ratios much larger than 10:1), the more massive galaxy will shred the satellite (e.g. White 1983, Holley-Bockelmann \& Richstone 1999), and the black hole will sink toward the central black hole without a large reservoir of gas. On the other hand, the merger of comparable (mass ratios less than 10:1) galaxies will generate ample gas accretion onto the central black hole, as the satellite triggers a central starburst (Mihos \& Hernquist 1994). Here we distinguish two cases, depending on the mass ratio of merging DMHs. The first is a more conservative criterion that allows black holes to accrete gas if the mass ratio of host DMHs is less than 4:1 (4:1 accretion). The second case sets an upper constraint on the mass of the final black hole by allowing seeds to accrete gas as long as the merging DMHs have a mass ratio less than 10:1 (10:1 accretion). In the following section, we compare all three growth scenarios: black hole growth through mergers only (dry growth); growth through mergers combined with 4:1 gas accretion (4:1 growth); and finally, growth through mergers combined with 10:1 gas accretion (10:1 growth). These are simplifying assumptions made to explore parametrically the range of solutions in our scenario.

\section{RESULTS}

From $\mathrm{z}=19$ to $\mathrm{z}=12$, we identified all of the DMHs in the manner described in the previous section. With this approach, we obtained the initial positions and formation redshifts for 1477 seed black holes, approximately 100 new MBHs per snapshot. We assigned an initial mass of $200 \mathrm{M}_{\odot}$ to each MBHs and traced their merger history from $0 \lesssim \mathrm{z} \lesssim 19$.

\subsection{Growth of Sagittarius A*}

Throughout the simulation, one DMH emerges as the largest in mass and dominates the dynamics of its group. Its mass at $\mathrm{z}=0$ is $\mathrm{M}_{\text {primary }}=4.5 \times 10^{12} \mathrm{M}_{\odot}$ which roughly corresponds to Andromeda's DMH, and is somewhat higher than estimates for the Milky Way's halo (Dehnen et al 2006). This halo grows through the smooth accretion of dark matter particles as well as DMH mergers. Meanwhile, the central black hole grows through gas accretion (if we included it) and mergers. We traced the growth of both the primary halo and its massive black hole as a function of redshift (Figure 1). Figure 1 compares the growth of both the black hole (dry) and its host DMH. Overplotted is a descriptive redshift dependence because it exists only where the curve has a negative slope. Initially, the primary halo grows in mass only through smooth accretion and reaches $\mathrm{M}_{\text {primary }}=10^{9} \mathrm{M}_{\odot}$ by $\mathrm{z}=15$ (Fig. 1). During this period, the black hole remains at its initial mass. At all other redshifts, the growth of primary halo and any other DMH can be described as a cycle of steady accretion of the surrounding dark matter punctuated by rapid growth through mergers with incoming DMHs.

We present the DMH merger rates in Figure 2. In comparing Figure 1 and Figure 2, notice that changes in merger rate catalyze both the $\mathrm{MBH}$ and primary halo mass growth. Figure 2 presents merger rates as the number of mergers per unit time per unit redshift for all halos, while Figure 1 traces the merger history for the primary halo only. Nevertheless, since the primary halo participates in $90 \%$ of all DMH mergers, Figure 2 essentially follows the evolution of the primary halo. At z 11 , the central black hole with mass $\mathrm{M}_{\mathrm{BH}} \sim 3000 \mathrm{M}_{\odot}$ has reached the IMBH range. At this redshift, the merger rate reaches maximum (Fig 2.). Consequently, the IMBH grows quickly from $\sim 3000 \mathrm{M}_{\odot}$ to $\sim 10,000 \mathrm{M}_{\odot}$ although surprisingly the bound DMH mass decreases 1 Merger rates decrease at $\mathrm{z} \lesssim 10$ but experience two more peaks with periods of violent mergers around $\mathrm{z}=6$ and $\mathrm{z}=3$ (Figure 2).

At $\mathrm{z}=0$, there is one $\mathrm{SMBH}$ with mass $\mathrm{M}_{\mathrm{SMBH}}=2.9 \times 10^{5} \mathrm{M}_{\odot}$ at the center of the primary halo (Figure 1 and Figure 3 thick line). This mass is too small to match present day observations, so we know, as has been stated, that mergers alone can not create a SMBH. As described before, we used the Salpeter approximation to model different gas accretion scenarios where the accretion efficiency depends on the mass ratio of merging DMHs. In all three scenarios, the black hole reaches the IMBH range at $\mathrm{z} \sim 12$ (Figure 3). As the number of DMH mergers increases, the IMBH continues growing - faster, naturally, if gas accretion is more efficient. In a 4:1 growth scenario, the IMBH grows to a $2.3 \times 10^{6} \mathrm{M}_{\odot}$ SMBH by $\mathrm{z}=5.5$. At these redshifts, low mass ratio mergers of DMHs are depleted and SMBH growth through gas accretion stops. Therefore, the duty cycle of the active galactic nucleus (AGN) hosting this $\mathrm{SMBH}$ dies at $\mathrm{z}=5.5$, too. In this model, the SMBH mass remains essentially constant for $\$ 5.5$, because it only experiences very high mass ratio mergers with $200-1000 \mathrm{M}_{\odot}$ black holes. Finally, at $\mathrm{z}=0$ the AGN has evolved into galaxy hosting a $2.3 \times 10^{6} \mathrm{M}_{\odot} \mathrm{SMBH}$ at its center, strikingly similar to the mass of the Milky Way SMBH (Schodel et al. 2002, Ghez et al. 2003, 2005). A highly efficient 10:1 growth scenario yields an upper constraint of $3.4 \times 10^{7} \mathrm{M}_{\odot}-$ comparable to the SMBH observed in M31 (Bender et al. 2005).

Figure 4 shows the SMBH mass as a function of central velocity dispersion and redshift for our three growth scenarios.

1 In DMH mergers, the outskirts can become unbound for a short period of time, but may be reaccreted as the structure grows. The large number of DMH mergers between $10 \lesssim z \lesssim 12$ (cf Figure 2), increases the number of unbound dark matter particles, since there is not enough time for reaccrete before another merger. 
We obtained the velocity dispersion for dark matter at $10 \mathrm{kpc}$ comoving from the halo center. Recall that $10 \mathrm{kpc}$ comoving corresponds to $700 \mathrm{pc}$ at $\mathrm{z}=19$ and $14 \mathrm{kpc}$ at $\mathrm{z}=0$. Similar to Figure 3, every decrease in dark matter velocity dispersion is a consequence of high merger rates at a specific redshift. The maximum value for the velocity dispersion obtains at $\mathrm{z}=0$, $\sigma=120 \mathrm{~km}^{-1}$. When compared to the M- $\sigma$ relation from Gebhardt et al. 2001 and Merritt \& Ferrarese 2001, a SMBH of $\sim 3 \times 10^{7} \mathrm{M}_{\odot}$ has a stellar velocity dispersion of $\sigma=120 \mathrm{~km}^{-1}$ at $\mathrm{z}=0$, which matches the SMBH mass in the 10:1 growth scenario. Of course our M- $\sigma$ relation is derived only from the dark matter and at much larger radii, but this may give us a hint that the M- $\sigma$ relation is tied to the dynamics of the global potential. Exploring the formation of more massive SMBHs under this set of assumptions, including the redshift at which growth occurs and the merger rate as a function of redshift is beyond the scope of this paper.

\subsection{Black Hole Merger Rates and ULXs}

Although there are no studies of AGN duty cycle for $\mathrm{z}>6$ and $\mathrm{M}_{\mathrm{SMBH}} \lesssim 10^{6} \mathrm{M}_{\odot}$ (Wang et al. 2006), it is probable that the AGN fueling mechanism - gas accretion - is similar to that of higher mass galaxies. The SMBH in 4:1 and 10:1 growth scenarios grows to its final mass of $\mathrm{M}_{\mathrm{SMBH}} \sim 10^{6} \mathrm{M}_{\odot}-f e w \times 10^{7} \mathrm{M}_{\odot}$ in the redshift range $5.5 \lesssim \mathrm{z} \lesssim 6$. As major mergers dictate the growth at $\mathrm{z}>6$, the SMBH evolves as a low luminosity AGN with the duty cycle governed by on and off switching of accretion onto the black hole. The AGN duty cycle drops to zero at $\mathrm{z} \sim 6$ as the host galaxy becomes too large for major mergers to occur and both the SMBH and its host galaxy enter a quiet phase of their growth through mergers with much smaller galaxies.

Interestingly, all incoming small galaxies at $\mathrm{z} \sim 6$ in our model carry an $\mathrm{IMBH} \sim 1000 \mathrm{M}_{\odot}$. This sets the stage for a phase where each new galaxy merger can be characterized as a dwarf starburst galaxy with a central IMBH accreting gas while sinking toward the SMBH at the center. In other words, in these later phases the lower mass incoming black hole may accrete gas, but we assume no significant gas accretion onto the central SMBH because of the high mass ratio of the black holes. With the exception of the largest black hole in our simulation, all other black holes fall into the IMBH range: $200 \mathrm{M}_{\odot} \lesssim \mathrm{M}_{\mathrm{IMBH}} \lesssim 10^{4} \mathrm{M}_{\odot}$. These black holes are of the special interest as candidates for ULX sources (Mii \& Totani 2005). ULXs are interpreted as massive black holes accreting gas in starburst galaxies, although this is still a matter of debate (King et al. 2001).

Figure 5 shows the merger rates for different mass ratio ranges and total binary masses. When deconstructed into ranges of mass ratios and $\log$ total binary mass (defined as $\mathrm{p}=\log \left(\mathrm{m}_{1}+\mathrm{m}_{2}\right)$ ), these merger rates can be used to predict the number of ULX sources in starburst galaxies throughout the galaxy evolution, assuming that merging galaxies are reasonably gas rich. For the mass ratio $1 \lesssim \mathrm{m}_{1} / \mathrm{m}_{2}<10$, we see only minor differences between the three growth scenarios for any range of binary mass (Fig 5a, 5b, 5c). For $\mathrm{z} \gtrsim 10$ black holes grow mostly through mergers. For $\mathrm{z} \sim 8$, mass ratio $<10$ and binary mass $10^{3} \mathrm{M}_{\odot}<\mathrm{m}_{\mathrm{BH}}<10^{4} \mathrm{M}_{\odot}(3 \lesssim \mathrm{p}<4)$ the merger rate is $\mathrm{R} \sim 10 \mathrm{yr}^{-1}$ in all three growth scenario - and it reaches a maximum $\mathrm{R}_{\max } \sim 30 \mathrm{yr}^{-1}$ for $\mathrm{p}<3$. At lower redshifts the merger rates decrease rapidly (Fig $5 \mathrm{a}, 5 \mathrm{~b}, 5 \mathrm{c}$ ), and gas accretion becomes important (Figure 3). Nevertheless, there are no changes in Fig $5 \mathrm{a}, 5 \mathrm{~b}, 5 \mathrm{c}$ for $\mathrm{z}<10$ since the mass ratio of merging black holes becomes very large as the higher mass black holes gain mass preferentially (Figure 6). In our scenario, only the black holes at the centers of merging DMHs grow through gas accretion; a large number of DMHs remain isolated for most of the simulation and merge late in high mass ratio encounters (middle and bottom panels of Fig 5 ). The difference between the top and bottom panels in Figure 5 shows that most of the black hole mergers at high redshifts are with $1 \lesssim \mathrm{m}_{1} / \mathrm{m}_{2}<10$ mass ratio.

Since black holes grow faster with more efficient gas accretion, the merger rates for mass ratio $10 \lesssim \mathrm{m}_{1} / \mathrm{m}_{2}<100$ and total binary mass $10^{4} \mathrm{M}_{\odot}<\mathrm{m}_{\mathrm{BH}}<10^{6} \mathrm{M}_{\odot}$, shift toward higher redshifts in our accretion models (Fig 5d, 5e, 5f). In this mass ratio range, $3 \lesssim p<4$ mergers peak at $8 \gtrsim z \gtrsim 10$, with a merger rate of $\sim 10$ depending on the growth scenario. Very high mass ratio mergers $10^{2} \lesssim \mathrm{m}_{1} / \mathrm{m}_{2}<10^{4}$ are of the special interest (Fig 5 . bottom panels). At $\mathrm{z}<6$, high mass ratio DMH mergers correspond to a dwarf galaxy or globular cluster being consumed by massive galaxy. The IMBH carried by smaller counterpart will eventually coalesce with SMBH at the center by first forming a binary with total mass $\mathrm{p}>5$ and high mass ratio $10^{2} \lesssim \mathrm{m}_{1} / \mathrm{m}_{2}<10^{4}$. This is an ideal LISA source, as will be explored in more detail in our next paper.

As a result, the merger rates in Figure 5d, 5e, $5 \mathrm{f}$ and Figure 7 for the above total binary mass and mass ratio ranges directly correspond to the number of IMBHs per galaxy per redshift. Since the accretion timescale is approximately half the merger time scale, the ULX number density is $\mathrm{N}_{\mathrm{ULX}}=\mathrm{R} / 2$. In the case of dry growth (Fig $5 \mathrm{~g}$ ), a black hole binary with mass $10^{5} \mathrm{M}_{\odot}<\mathrm{m}_{\mathrm{BH}}<10^{6} \mathrm{M}_{\odot}$ has an approximately constant and high merger rate of $\mathrm{R} \sim 15 \mathrm{yr}^{-1}$ for $2<\mathrm{z}<6$. This corresponds to $\sim 7$ ULX sources per starburst galaxy at $2<\mathrm{z}<6$. Similarly the $10^{5} \mathrm{M}_{\odot}<\mathrm{m}_{\mathrm{BH}}<10^{6} \mathrm{M}_{\odot}$ binary in the 4:1 growth scenario has $\mathrm{R} \lesssim 10 \mathrm{yr}^{-1}$ (Fig $5 \mathrm{~h}$ ) and $\mathrm{N}_{\mathrm{ULX}}=5$. More importantly Figure $5 \mathrm{~h}$ shows $\mathrm{N}_{\mathrm{ULX}}>2$ at $2<\mathrm{z}<10$. For even higher mass ratios $10^{4} \lesssim \mathrm{m}_{1} / \mathrm{m}_{2}<10^{5}$, the merger rate is $\mathrm{R} \lesssim 12 \mathrm{yr}^{-1}$ for $\mathrm{z} \lesssim 3$.

Figure 7 shows merger rates for the $10: 1$ growth scenario and high mass ratio $10^{4} \lesssim \mathrm{m}_{1} / \mathrm{m}_{2}<10^{6}$ for $10^{6} \mathrm{M}_{\odot}<\mathrm{m}_{\mathrm{BH}}<$ $10^{8} \mathrm{M}_{\odot}$ mergers. ULX sources in Figure 7 and Figure $5 \mathrm{~g}, 5 \mathrm{~h}$ have constant number density of $\sim 10$ per starburst galaxy at $\mathrm{z}>2$. The ULX number density drops rapidly at $\mathrm{z}=2$. Note that these ULX sources are not the same as the ULXB sources seen in local universe with much shorter accretion lifetimes. 
Miroslav Micic, Kelly Holley-Bockelmann, Steinn Sigurdsson, Tom Abel

\subsection{Comparison to Results from Press-Schechter theory}

We compare our results with those obtained by various black hole merger models from EPS theory. Figure 8 shows the merger rates for four Press-Schechter models decribed in Sesana et al. 2007. In the VHM model, massive DMHs $\left(\mathrm{M}_{\mathrm{DMH}}=10^{11}-10^{15}\right.$ $\left.\mathrm{M}_{\odot}\right)$ are seeded with $\mathrm{m}_{\mathrm{BH}} \sim 200 \mathrm{M}_{\odot}$ black holes at $\mathrm{z}=20$; in the KBD model, low mass halos $\left(\mathrm{M}_{\mathrm{DMH}}=10^{6}-10^{7} \mathrm{M}_{\odot}\right)$ are seeded with $\mathrm{m}_{\mathrm{BH}} \sim 5 \times 10^{4} \mathrm{M}_{\odot}$ at $15 \lesssim \mathrm{z} \lesssim 20$; and the BVR models explore different redshift ranges for seeding black holes in halos: $\mathrm{m}_{\mathrm{BH}}=10^{4}-10^{5} \mathrm{M}_{\odot}$ at $15 \lesssim \mathrm{z} \lesssim 20$ in the BVRhf model and $18 \lesssim \mathrm{z} \lesssim 20$ in the BVRlf model. Figure 8a shows superimposes their merger rates with our results. Since our numerical simulations have smaller mass resolution than the VHM model, their DMHs (and therefore black holes) start merging later with a merger rate peaking at $\mathrm{z}=12$. Our maximum merger rate is also higher because our range of seeding redshifts is larger $12 \lesssim \mathrm{z} \lesssim 19$. The mergers in question are small mass ratio mergers, with $\mathrm{m}_{\mathrm{BH}} \lesssim 10^{4} \mathrm{M}_{\odot}$ (Figure $8 \mathrm{~b}$ ). At $\mathrm{z} \lesssim 10$ our merger rates match the KBD model. However, we have multiple peaks at $\mathrm{z}=6$ and $\mathrm{z}=3$ from episodes of violent DMH dynamics that is not depicted by Press-Schechter theory (Figure 8a). This is an advantage of numerical simulations, one that acts to increase the merger rate, the black hole growth rate, and the mass ratio of typical mergers drastically.

In order to achieve $10^{4} \mathrm{M}_{\odot} \lesssim \mathrm{m}_{\mathrm{BH}} \lesssim 10^{6} \mathrm{M}_{\odot}$ (Figure 8c) with Press-Schechter models, one must start at higher redshifts and assume higher initial black hole masses. The KBD model starts with high mass seeds, and as a result the KBD merger rates peak for $10^{4} \mathrm{M}_{\odot} \lesssim \mathrm{m}_{\mathrm{BH}} \lesssim 10^{6} \mathrm{M}_{\odot}$, and are larger than the N-body rates, both in this mass range and at $\mathrm{z}>5$. We "spend" BH seeds naturally during mergers so in this mass range our peak is $\mathrm{m}_{\mathrm{BH}} \lesssim 10^{4} \mathrm{M}_{\odot}$ at $\mathrm{z}>5$.

\section{DISCUSSION}

We used a high-resolution cosmological N-body simulation to study the formation and growth of seed black holes into SMBHs and derived black hole merger rates. We used physically-motivated formalisms for seeding DMHs with black holes. Better understanding of the initial mass function for Population III black holes will improve the accuracy of our results in the future. Of course, black hole seeds do not necessarily have to be Population III black holes. We can use our approach to test different seeding scenarios. For example, it can be used to set constraints for primordial black hole formation (Mack et al. 2006).

Depending on the assumed growth scenario, we showed that gas accretion combined with hierarchical mergers of massive black holes leads to the formation of a Sagittarius A* type black hole in the center of a Milky Way-sized DMH. In the case of very efficient gas accretion, an M31-sized SMBH can form. In both cases, the SMBH reaches its final mass at z 6 . We showed that $\mathrm{z}=6$ may be a critical redshift for the transition from the AGN duty cycle dominated by high mass ratio DMH mergers to a starburst galaxy phase where low mass ratio DMH mergers supply a galaxy with a constant population of ULXs up to z=2. We argue that the $\mathrm{z}=6$ turning point is consistent with the maximum of AGN luminosity function at $\mathrm{z}=2$ since the SMBHs in our simulation have masses and luminosities below the currently observable range. Also note that potential ULX sources in our simulation are at $\mathrm{z}>2$. They may correspond to nuclear clusters at the centers of dwarf galaxies, and therefore likely sites of IMBHs formation. This population is different from the low redshift ULX population which may form from globular cluster dynamics and are likely undergoing short lived binary accretion.

The final stages of the black hole merger are followed by an emission of gravitational radiation, low in frequency but relatively high in amplitude. In one of our follow up papers, for each binary black hole inspiral and merger, the expected gravitational wave signal for the LISA will be determined, and the LISA event rate as a function of time calculated. In particular, we will study whether LISA observations will be able to distinguish between different assembly scenarios. One interesting source we will be able to constrain will be IMBH/SMBH mergers. We will calculate LISA detectability of IMBH mergers for different mass ranges and binary mass ratios. At this point, rough estimates can be made from Figure 4, considering the known ranges for LISA sensitivity. In case of dry growth (Fig $4 \mathrm{~g}$ ), a black hole binary with mass $10^{5} \mathrm{M}_{\odot}<\mathrm{m}_{\mathrm{BH}}<10^{6} \mathrm{M}_{\odot}$ is in the LISA range with a very high merger rate of $\sim 15 \mathrm{yr}^{-1}$. Interestingly, just as SMBHs dim electromagnetically at $\mathrm{z}=6$, they turn on in the gravitational wavebands.

There are a number of processes that might suppress massive black hole merger rates. We assumed that every first star will produce black hole as opposed to neutron star or pair detonation with no remnant. We also assume that black holes merge efficiently and that recoil ejection which is a function of spin, orientation, eccentricity, and mass ratio of merging black holes is negligible. All of these processes will be addressed in the follow up paper.

Finally, since our $O\left(10^{6}\right) \mathrm{M}_{\odot}$ black hole was in place so early, it might be tempting to interpret our results as an indictment against downsizing, the recent observation that the number density of low mass AGNs peaks at $z<1$ (Cowie et al. 2003, Merloni 2004, Heckman et al. 2004). However, we caution that in order to achieve such high mass resolution, we simulated a small volume, and are therefore plagued by small number statistics. In fact, if we were to scale our simulation up in mass, so that the most massive SMBHs were $O\left(10^{10}\right) \mathrm{M}_{\odot}$, we find some indication that the more isolated DMHs will host low mass SMBHs that gain most of their mass at late redshifts compared to the most massive SMBH. We plan to explore the growth of black holes as a function of environment and smooth accretion efficiency in a follow up paper. 
Supermassive Black Hole Growth and Merger Rates from Cosmological N-body Simulations

\section{ACKNOWLEDGMENTS}

We would like to thank NASA's Columbia High End Computing program for a generous time allocation, and the Center for Gravitational Wave Physics at Pennsylvania State University for sponsoring this research. This research was supported by a grant from the NSF, PHY 02-03046 and from NASA's ATP NNG04GU99G. SS would also like to thank KITP at UCSB and SLAC at Stanford University for their hospitality.

\section{REFERENCES}

Abel, T., Bryan, G., \& Norman, M., 2000, ApJ, 540, 39

Abel, T., Bryan, G., \& Norman, M., 2002, Sci, 295, 93A

Bender, R., Kormendy, J., Bower, G., 2005, ApJ, 631, 280

Berczik, P., Merritt, D., Spurzem, R., Bischof, H.P., \& 2006, ApJ, 642L, 21B

Cowie, L.L., Barger, A.J., Bautz, M.W., \& Brandt, W.N., Garmire, G.P., 2003, ApJ, 584, L5

Davis, M., Efstathiou, G., Frenk, C., White, S.D.M., \& 1985, ApJ, 292, 371

Dehnen, W., McLaughlin, D.E. \& Sachania, J., 2006, MNRAS, 369, 1688

Erickcek, A.L., Kamionkowski, M., \& Benson, A.J., 2006, MNRAS, tmp, 940E

Escala, A, Larson, R.B., Coppi, P.S., \& Mardones, D., 2005, ApJ, 630, 152E

Fabbiano, G., 1989, ARA\&A, 27, 87

Fabbiano, G., White, N.E., 2006, \& in Compact Stellar X-Ray Sources, ed. W. H. G. Lewin \& M. van der Klis \& (Cambridge: Cambridge Univ. Press), 475

Gao, L., White, S.D.M., Jenkins, A., Frenk, C.S., Springel, V., \& 2005, MNRAS, 363, 379

Gehz, A.M., Duchene, G., Matthews, K., 2003, ApJ, 586, 127

Gehz, A.M., Salim, S., Hornstein, S.D., 2005, ApJ, 620, 744

Haehnelt, M.G., 1994, MNRAS, 269, 199 Milosavljevic, M., ApJL, submitted, astro-ph/0306074

Heckman, T.M., Kauffmann, G., Brinchmann, J., \& Charlot, S., Tremonti, C., White, S.D.M., 2004, ApJ, 613, 109

Heger et al., 2003, 2003, ApJ, 591, 288H

Holley-Bockelmann, K., \& Sigurdsson, S., 2006, astro-ph, 1520H

Holley-Bockelmann, K., \& Richstone, D.O., 1999, ApJ, 517, 92H

Islam, R. R., Taylor, J. E., Silk, J., \& 2003, MNRAS, 340, 647I

King, A.R., Davies, M.B., Ward, M.J., Fabbiano, G. \& Elvis, M., 2001, ApJL, 552, 109

Kormendy, J., Richstone, D., 1995, ARA\&A, 33, 581K

Lacey, C., Cole, S., 1993, MNRAS, 262, 627

Machacek, M.E., Bryan, G.L., \& Abel, T. 2001, ApJ, 548, 509

Mack, K.J., Ostriker, J.P., Ricotti, M., astro-ph/0608642

Menou, K., Haiman, Z., Narayanan, V.K., \& 2001, ApJ, 558, 535

Merloni, A., 2004, MNRAS, 353, 1035

Merritt, D., Ferrarese, L., 2001, ApJ, 547, 140M

Mihos, J.C., Hernquist, L., 1994, ApJ, 425L, 13M

Mii, H., Totani, T., 2005, ApJ, 628, 873

Micic, M., Abel, T., Sigurdsson, S., 2006, MNRAS, 372, 1540M

Nagashima, M., et al. 2005, ApJ, 634, 26N

Press, W.H., Schechter P., 1974, ApJ, 187, 425

Ptak, A., Colbert, E., 2004, ApJ, 606, 291

Reed, D.S., Bower, R., Frenk, C.S., Jenkins, A, Theuns, T., \& 2007, MNRAS, 374, 2R

Rhook, K.J., Wyithe, J.S.B., 2005, MNRAS, 361, 1145

Roberts, T., Warwick, R., 2000, MNRAS, 315, 98

Sesana, A., Haardt, F., Madau P., \& Volonteri, M., 2004, ApJ, 611, 623

Sesana, A., Volonteri, M., Haardt, F., \& 2007, astro-ph/0701556

Schneider, R., Ferrara, A., \& Natarajan, P., Omukai, K., 2002, ApJ, 571, 30

Schodel, R., Ott, T., Genzel, R., 2002, Nature, 419, 694

Sigurdsson, S., 2003, CQGra, 20S, 45S

Springel, V., Yoshida, N., \& White, S. D. M., 2001, NewA, 6, 79

Springel, V., 2000, MPA

Volonteri, M., Haardt, F., \& Madau, P., 2003, ApJ, 582, 559

Wang, J.M., Chen, Y.M., Zhang, F., 2006, ApJ, 647L, 17W

White, S.D.M., 1983, ApJ, 274, 53W

Wise, J., H., Abel, T., 2005, ApJ, 629, 615W

Wyithe, J.S.B., Loeb, A., 2003, ApJ, 590, 691 


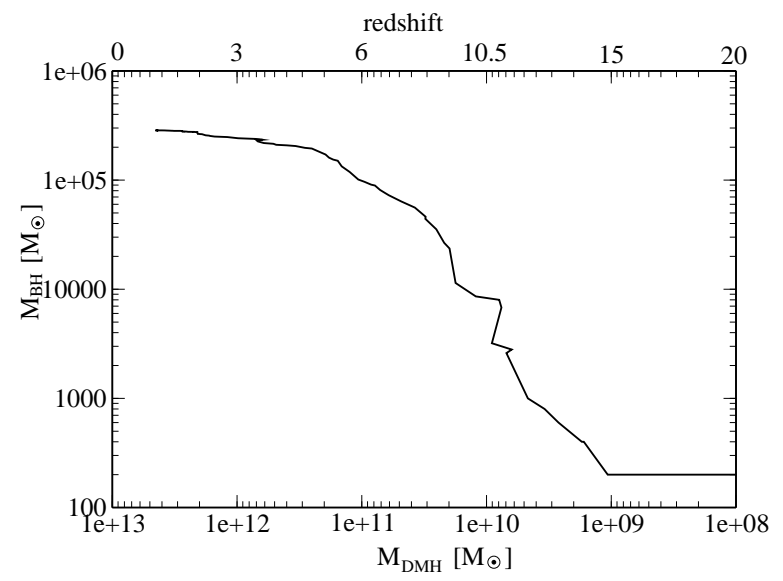

Figure 1. Mass of the SMBH at the center of primary halo as a function of primary halo's mass and redshift in the dry growth scenario. The growth of primary halo and any other DMH can be described as a cycle of steady accretion of the surrounding dark matter followed by rapid growth through mergers with incoming DMHs. This process is best observed at $\mathrm{z}=11$.

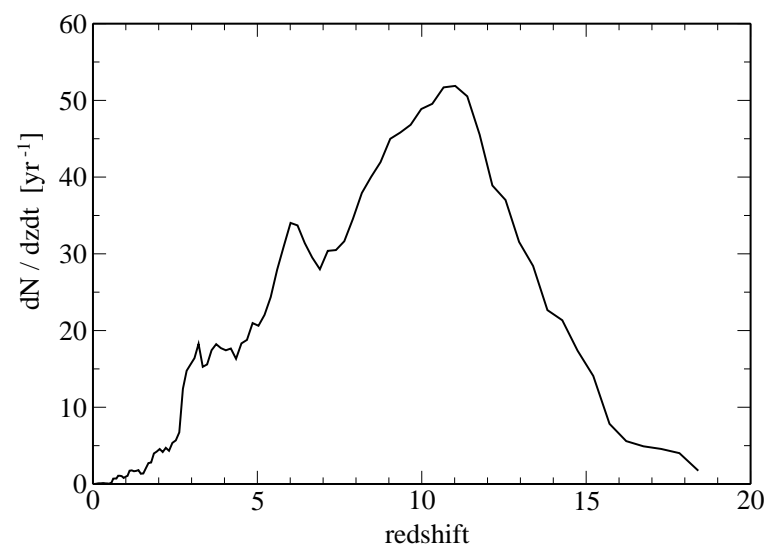

Figure 2. Merger rates per unit time per unit redshift observed at $\mathrm{z}=0$ as a function of redshift. Merger rates reach maximum at $\mathrm{z}=11$, decrease at redshifts $\mathrm{z} \lesssim 10$ but experience two more peaks with periods of violent mergers around redshifts $\mathrm{z}=6$ and $\mathrm{z}=3$. 


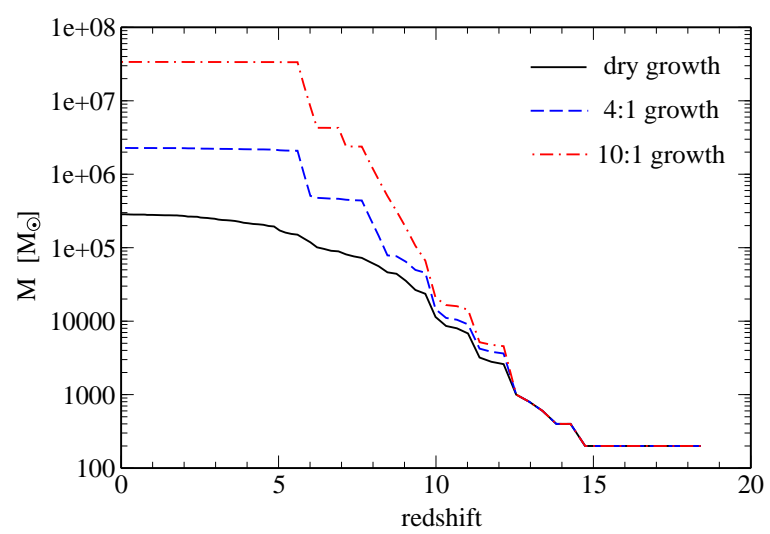

Figure 3. Growth of SMBH in three different accretion scenarios presented as mass of the SMBH as a function of redshift. Black hole mergers are dominant way of growing IMBH at $\mathrm{z} z 12$. Gas accretion is important at $6 \lesssim z \lesssim 12$. At redshift $\mathrm{z}=6$, SMBH reaches the maximum mass marking the transition of its host AGN into the starburst galaxy.

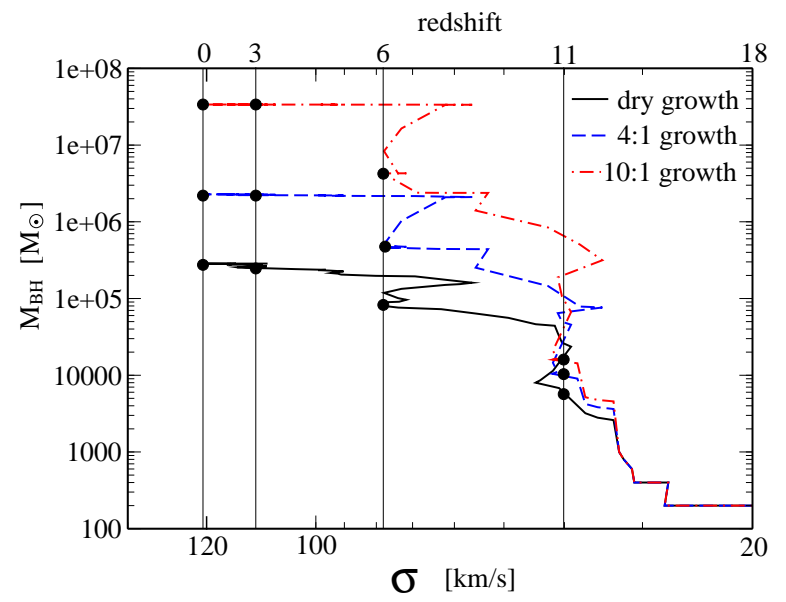

Figure 4. Growth of SMBH in three different accretion scenarios presented as mass of the SMBH as a function of dark matter central velocity dispersion at $\mathrm{r}=10 \mathrm{kpc}(\sigma)$, and as a function of redshift. Redshift dependence is descriptive hence filled circles correspond to data points in terms of redshift. $\sigma=120 \mathrm{~km}^{-1}$ at $\mathrm{z}=0$ and it fits the M- $\sigma$ relation for 10:1 growth accretion. 


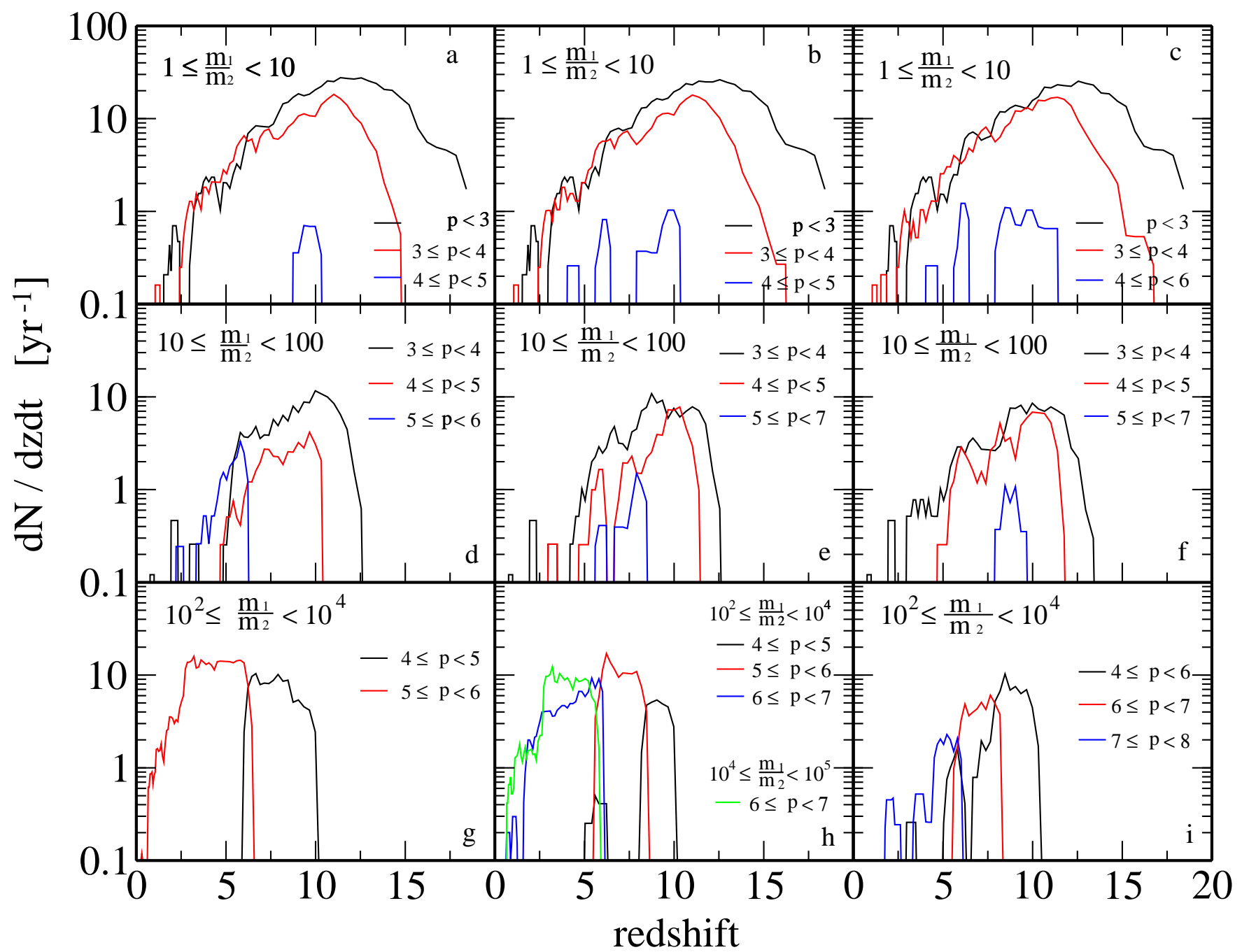

Figure 5. IMBH merger rates as observed at $\mathrm{z}=0$ as a function of redshift and presented for different binary mass ratios and total binary mass ranges, $\mathrm{p}=\log \left(\mathrm{m}_{1}+\mathrm{m}_{2}\right) .1 \lesssim \mathrm{m}_{1} / \mathrm{m}_{2}<10$ in $\mathrm{a}, \mathrm{b}, \mathrm{c} ; 10 \lesssim \mathrm{m}_{1} / \mathrm{m}_{2}<100$ in $\mathrm{d}$, e, f; $100 \lesssim \mathrm{m}_{1} / \mathrm{m}_{2}<10000$ in $\mathrm{g}$, h, i; dry growth in a, d, g; 4:1 growth in b, e, h; 10:1 growth in c, f, i. Most of the mergers are low mass ratio mergers at $\mathrm{z}>10$. However, high mass ratio mergers at $\mathrm{z}<10(\mathrm{~g}, \mathrm{~h})$ are in the LISA range and have large merger rates $\mathrm{R} \sim 15 \mathrm{yr}^{-1}$ for a wide range of redshifts $2<\mathrm{z}<10$. 


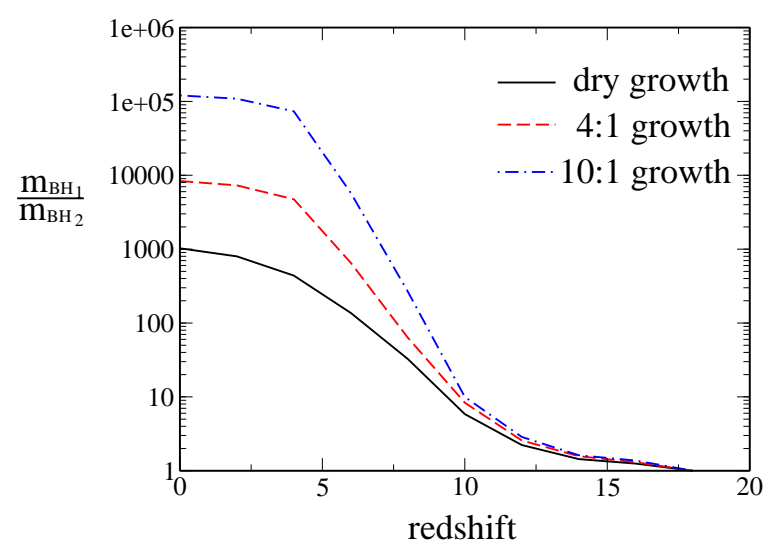

Figure 6. Mass ratio of merging black holes averaged over all mergers at a specific redshift for all three growth models. Black holes grow mostly through mergers at $\mathrm{z}>10$. Differences between three growing models become apparent at $\mathrm{z}<10$ where the increase in mass ratio of merging black holes is due to gas accretion.

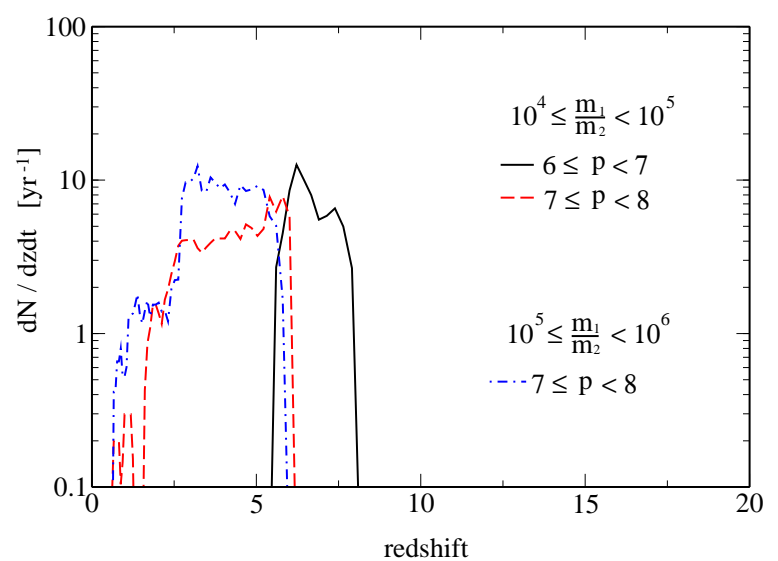

Figure 7. Merger rates observed at $\mathrm{z}=0$ as a function of redshift, presented is the case of 10:1 growth scenario and merger rates for large binary mass and extreme binary mass ratios, $\mathrm{p}=\log \left(\mathrm{m}_{1}+\mathrm{m}_{2}\right)$. Similar to Figure $5 \mathrm{~g}$ and $5 \mathrm{~h}$, mergers of SMBH with IMBH at $\mathrm{z}$ $>2$ will be observed by LISA, $\mathrm{R} \sim 10 \mathrm{yr}^{-1}$. 


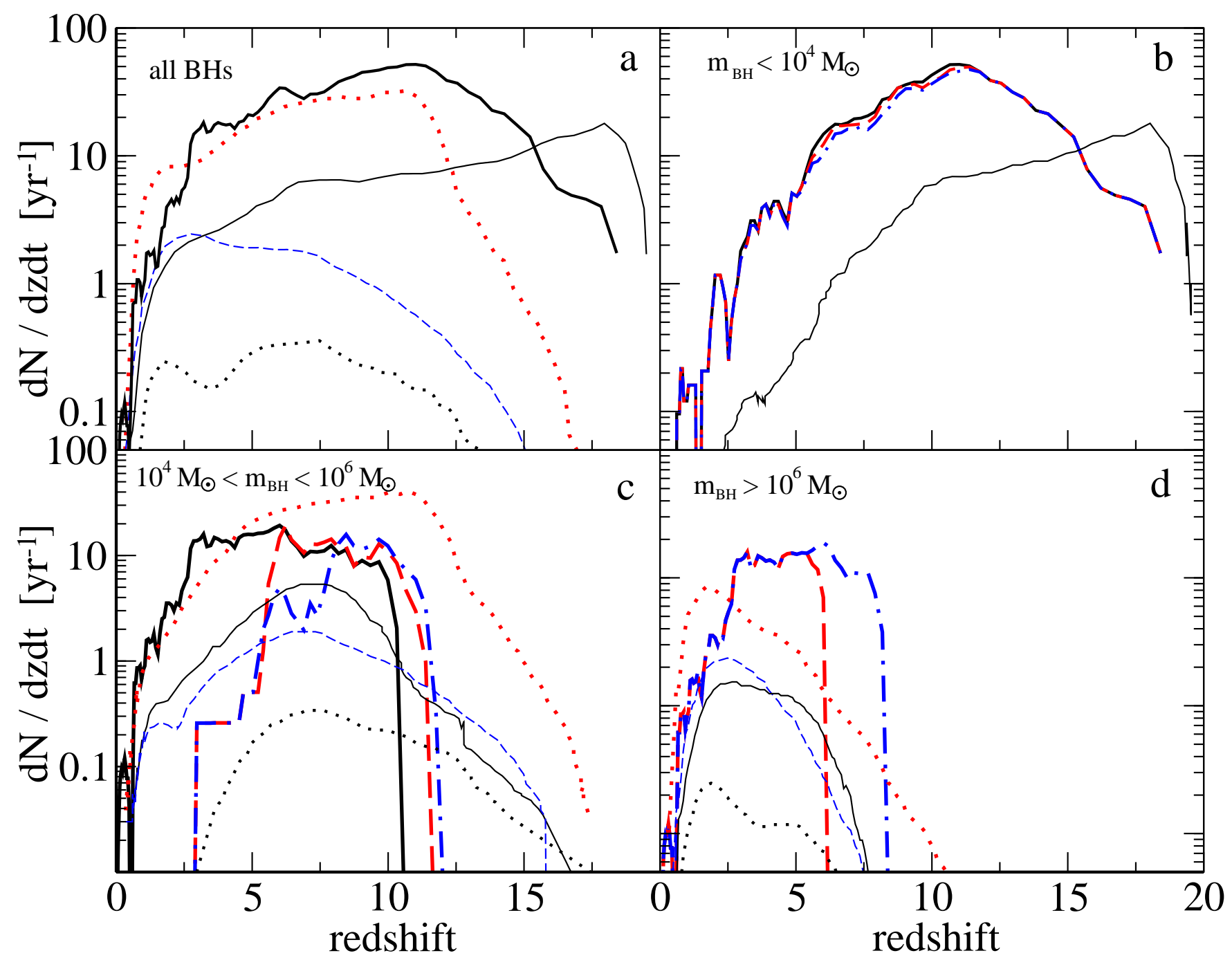

Figure 8. Merger rates observed at $\mathrm{z}=0$ as a function of redshift in different binary mass intervals $\mathrm{m}_{\mathrm{BH}}=\mathrm{m}_{1}+\mathrm{m}_{2}$ for four models described in Sesana et al. 2007, VHM in thin black line; KBD in dot-red line; BVRlf in blue-dashed line and BVRhf in black-dot line. Overplotted are merger rates from our N-body simulations for dry growth in thick black line; 4:1 growth in red dashed line and 10:1 growth in blue dashed-dot line. We predict massive black hole merger rates to be larger than those predicted by semi-analitical models in the range $2<\mathrm{z}<15$. Numerical simulations also depict episodes of violent DMH dynamics which can be seen in multiple peaks at $\mathrm{z}=11 ; \mathrm{z}=6$; and $\mathrm{z}=3$. 